\title{
128 Bit Low Power and Area Efficient Carry Select Adder
}

\author{
Sudhanshu Shekhar \\ Pandey \\ School of Electronics \\ Engineering. \\ VIT University, Vellore-632014, \\ India
}

\author{
Amit Bakshi \\ School of Electronics \\ Engineering. \\ VIT University, Vellore-632014, \\ India
}

\author{
Vikash Sharma \\ Assistant Professor \\ BBDIT, Duhai, Ghaziabad \\ (U.P.) \\ India
}

\begin{abstract}
Carry Select Adder (CSLA) which provides one of the fastest adding performance. Traditional CSLA require large area and more power. Recently a new CSLA adder has been proposed which performs fast addition, while maintaining low power consumption and less area. This work mainly focuses on implementing the 128 bit low power and area efficient carry select adder using $0.18 \mu \mathrm{m}$ CMOS technology. Based on the efficient gate level modification, 128-b Square Scheme Block (SSB) CSLA) architecture have been developed and compared with the regular SSB CSLA architecture. The performance of the proposed SSB CSLA evaluated manually in terms of delay, power, and area manually with logical effort and also through custom design. The proposed design has been developed using verilog HDL and synthesized in cadence RTL compile using typical library of TSMC $0.18 \mu \mathrm{m}$ technology.
\end{abstract}

\section{Keywords}

CSLA, SSB CSLA, Area-Efficient, Low Power, Application Specific Integrated Circuit(ASIC)

\section{INTRODUCTION}

When we draw a block diagram or circuit diagram we define an input or output. However fast hardware is the gates or other things we have inside the circuit there will be a finite delay in the transmission of a signals this time is defined as propagation delay, of course depends on the length of the signal path as soon as the gates start switches transmission starts. When we want to design the fast circuit or fast system naturally we have to go for some solutions. By reducing the path of the transmission if we reduce the path so that we can reduce the delay and can increase the operation of the circuit. For the given technology we want to maximize the speed then we want to go for this type of scheme by cutting the short length. In the full adder circuit carry has to travel from state to state. Previous states carry need to require for the present state to do the operation. So, naturally when we increase the number of bits the propagation delay and the delay of each stage increases. Now if we don't have to depend on the transmission of the carry we can predict the carry of each stage. Now a day our computers speed is fast high in terms of GHz. So conceptually we need to improve the speed for the given design by decreasing several numbers of stage or gates. SSB Carry Select Adder (CSLA) has a more balanced delay, and requires lower power and area [1], [4],[8]. The basic idea of this work is to use Binary to Excess-1 Converter (BEC) instead of RCA with in the regular CSLA to achieve lower area and power consumptions [1],[2],[6],[7]. However the CSLA needs more area because of using multiples of Ripple Carry Adder for generating sum and carry on the dependency of carry input $\mathrm{Cin}=0$ and $\mathrm{Cin}=1[5]$. Then the final results of sum and carry are selected by the multiplexers from bit to bit going to increase. Finally reliable results at the output will depend upon the number of stages.

\subsection{BEC:}

As stated above the main idea of this work is to use BEC instead of the RCA with $\mathrm{C}=1$ in order to reduce the area and power consumption of the regular CSLA. To replace the n-bit RCA, an $\mathrm{n}+1$ bit $\mathrm{BEC}$ is required. A structure of a 4-bit BEC are shown in Fig. 1.

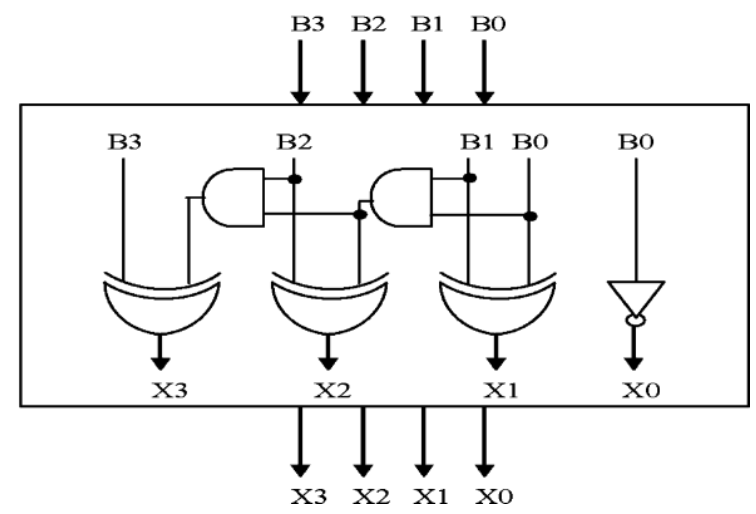

Fig 1: 4-b BEC.[1]

The basic function of the CSLA is obtained by using the 4-bit $\mathrm{BEC}$ together with the mux. One input of the 8:4 mux gets as it input (B3, B2, B1, and B0) and another input of the mux is the BEC output [1]. This produces the two possible partial results in parallel and the mux is used to select either the BEC output or the direct inputs according to the control signal Cin. The importance of the BEC logic stems from the large silicon area reduction when the CSLA with large number of bits are designed. The Boolean expressions of the 4-bit BEC is listed as (note the functional symbols NOT, \& AND, XOR)

$\mathrm{X} 0=\sim \mathrm{B} 0$

$\mathrm{X} 1=\mathrm{B} 0 \wedge$ B 1

$\mathrm{X} 2=\mathrm{B} 2 \wedge(\mathrm{B} 0 \& \mathrm{~B} 1)$

$\mathrm{X} 3=\mathrm{B} 3 \wedge(\mathrm{B} 0 \& \mathrm{~B} 1 \& \mathrm{~B} 2)$ 


\section{Evaluation methodology to calculate delay and area of the required basic adder blocks:}

The physical structure (AOI) of the Exor gate and numerical value represents the delay occurred by each of the individual gates. Each gate have delay equal to 1 unit and area equal to 1 unit [1]. The area evaluation has to be done by counting each of the AOI gates which will be required for implementing each logic blocks. The structure of the 128-bit regular SSB CSLA is shown in Fig. 2. It has 16 groups of different size RCA. The delay and area evaluation methodology of each group of 16-b SSB CSLA have been discussed in reference paper [1] and for 128-b manually calculated delay and area has shown in Table 1.

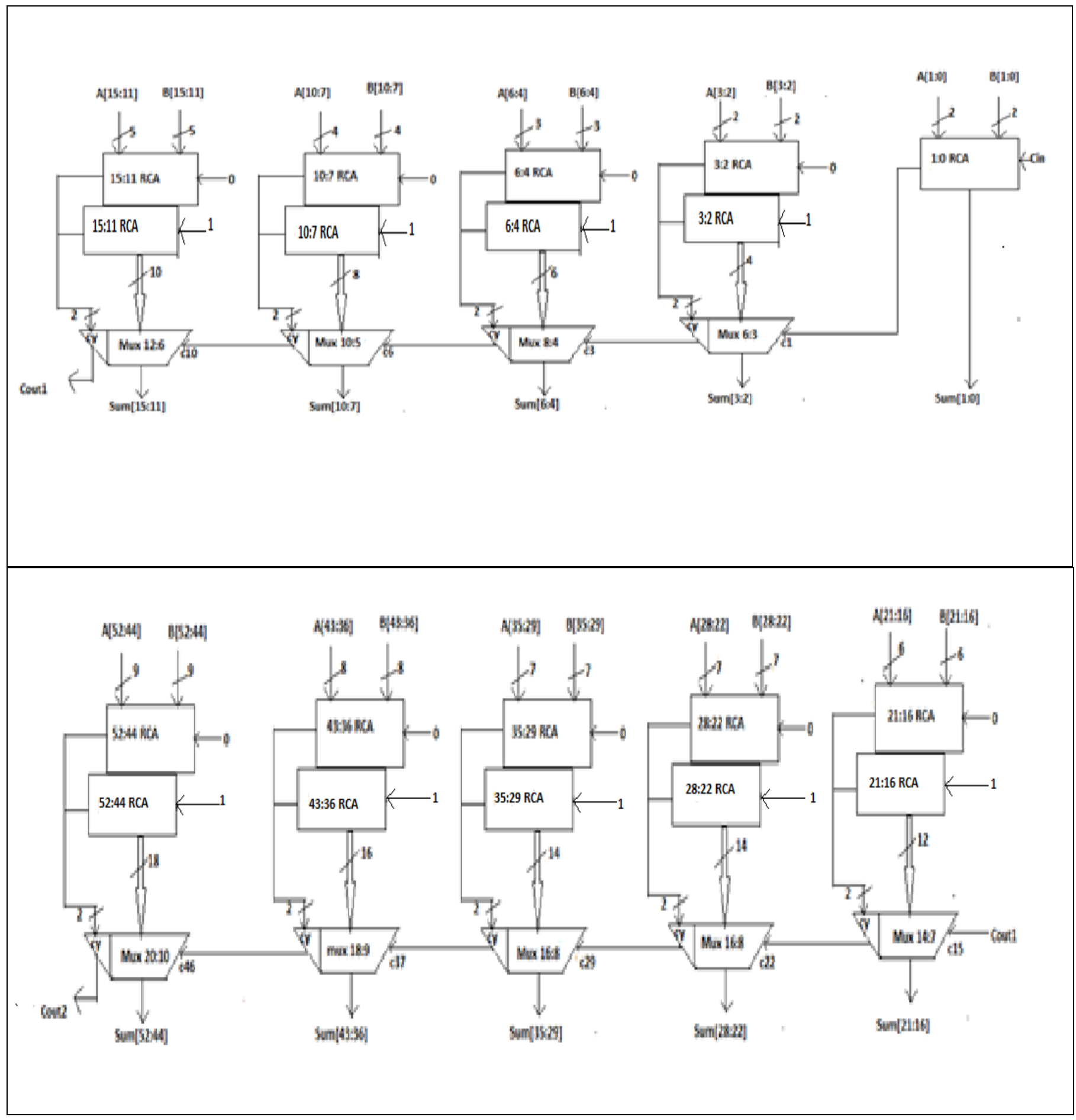




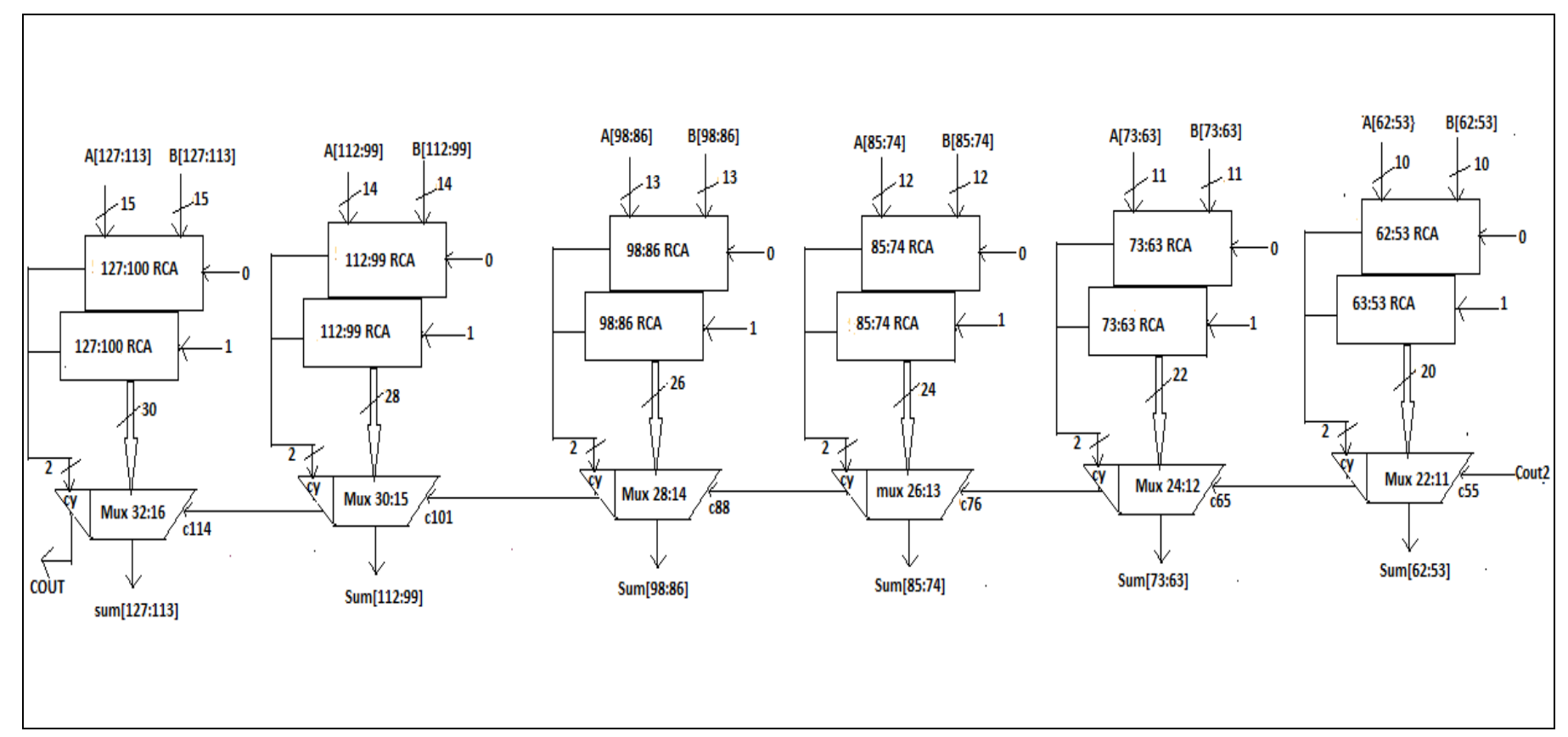

Fig 2: Regular 128-bit SSB CSLA

\subsection{Delay Evaluation Methodology Of Modified 128-Bit SSB CSLA:}

The main idea of this work is to use BEC instead of RCA with Cin=1 so that it can reduce power and area as compare with the regular CSLA. The structure of a 4 bit BEC are shown in the fig1.

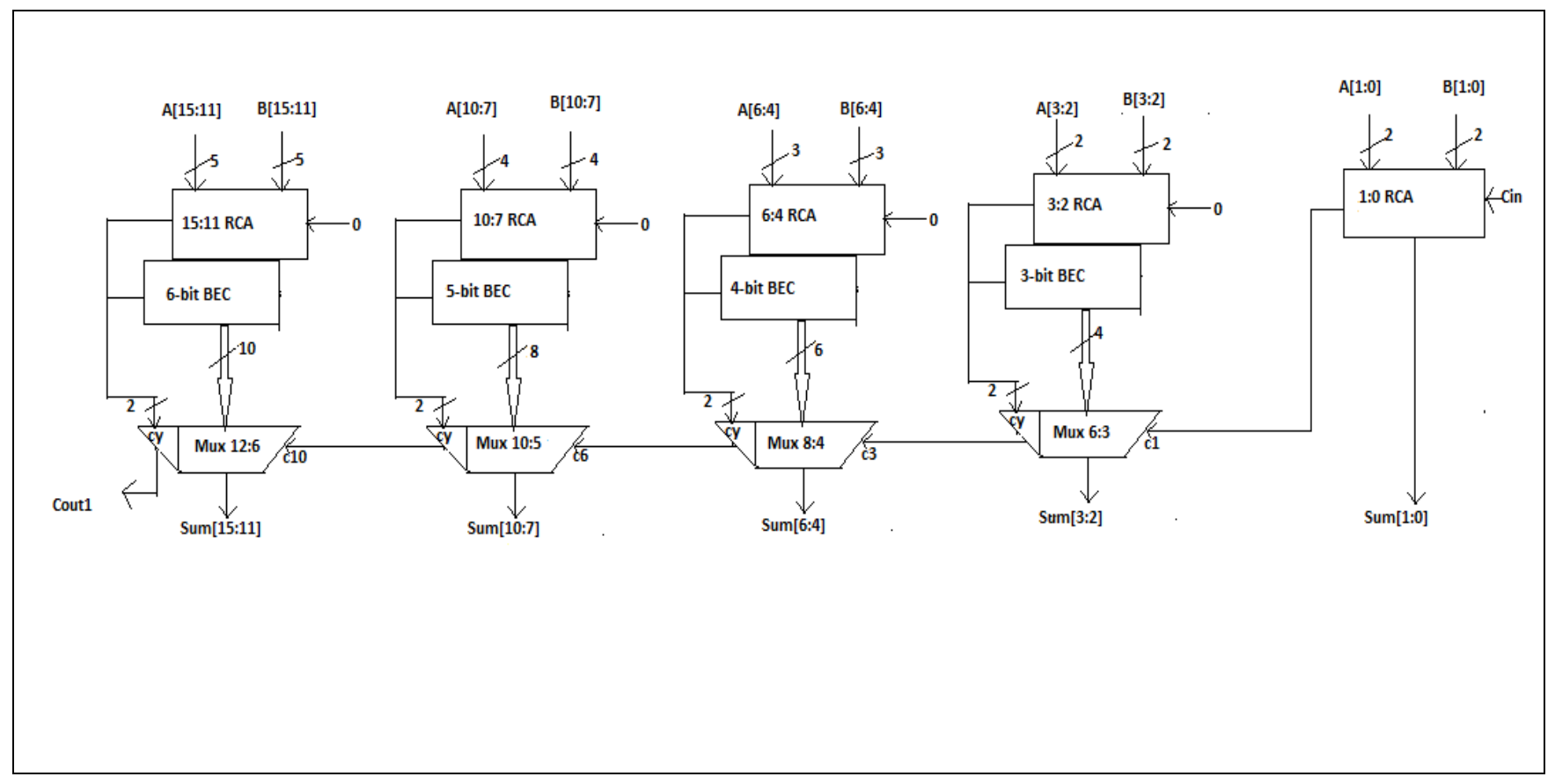




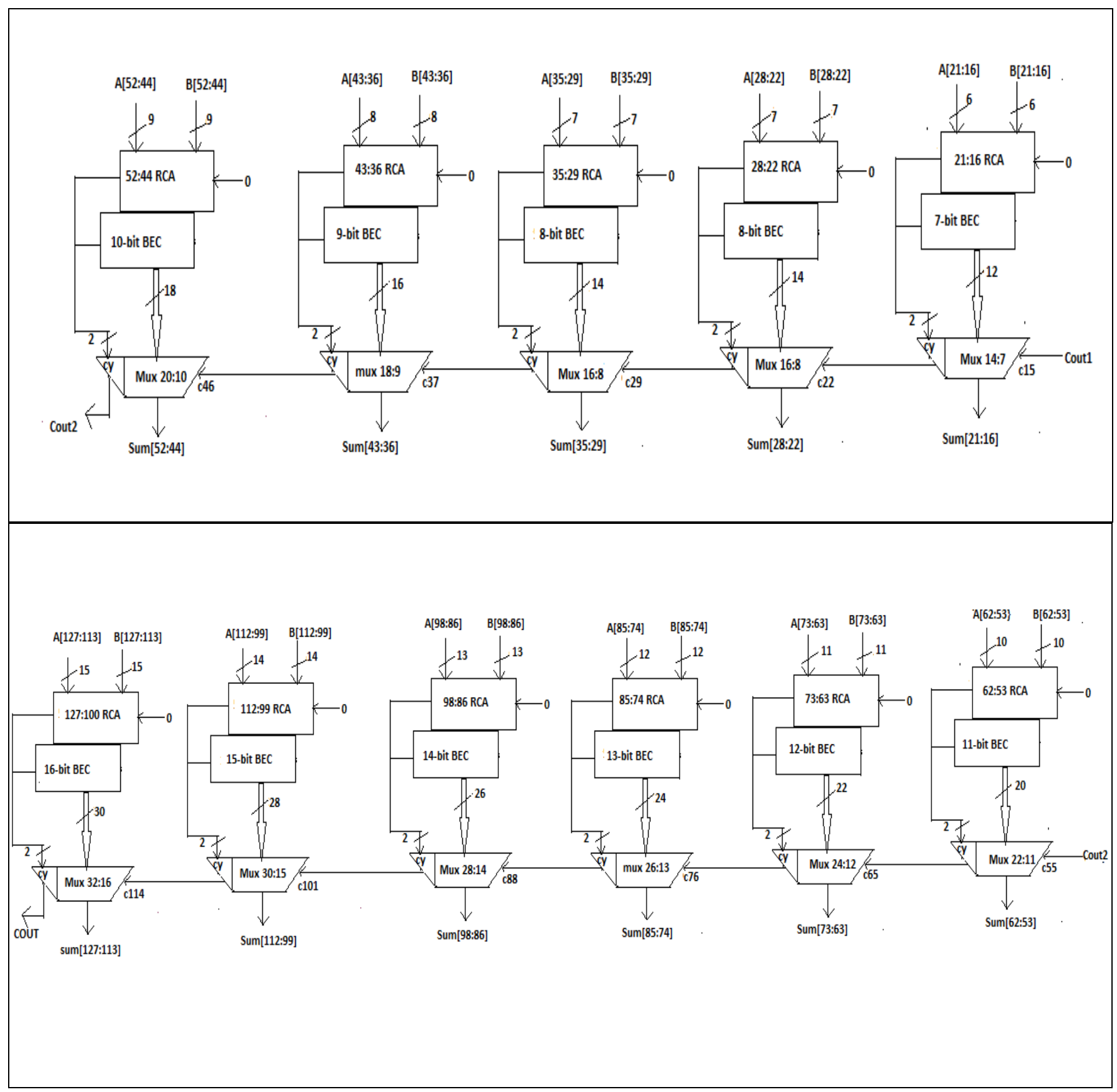

Fig 3: 128- bit Modified SSB CSLA

The structure of the modified 128-b SSB CSLA using BEC for RCA with Cin $=1$ to optimize the area and power is shown in Fig. 3. We again split the structure into 16 groups. The manually calculated delay and area estimation of group are shown in Table 1.

\section{ASIC Implementation Results:}

The design implemented in this paper has been developed using the verilog HDL and synthesized in Cadence RTL compiler using typical libraries of TSMC 0.18 um technology [3]. In this section we proposed an idea to reduce area, power and the timing slack of SSB CSLA architecture. We have compare the modified 128-bit SSB CSLA with the regular 128-bit CSLA and get the conclusion that by reducing number of gates we can reduce the area and the power. The percentage reduction of the area of the modified 128-bit SSB CSLA over regular SSB CSLA is $15.48 \%$. Whereas the total power reduction is $7.41 \%$.This work offer a great advantage in the reduction of area and also the total power. 
Table 1. Comparison of the manually calculated Delay and Area of the regular and Modified SSB CSLA

\begin{tabular}{|l|l|l|l|l|}
\hline Group & $\begin{array}{l}\text { Delay of } \\
\text { SSB } \\
\text { CSLA }\end{array}$ & $\begin{array}{l}\text { Delay of } \\
\text { Modified } \\
\text { SSB } \\
\text { CSLA }\end{array}$ & $\begin{array}{l}\text { Area of } \\
\text { SSB } \\
\text { CSLA }\end{array}$ & $\begin{array}{l}\text { Area of } \\
\text { Modified } \\
\text { SSB } \\
\text { CSLA }\end{array}$ \\
\hline Group2 & 11 & 13 & 57 & 43 \\
\hline Group3 & 13 & 16 & 87 & 61 \\
\hline Group4 & 16 & 19 & 117 & 84 \\
\hline Group5 & 19 & 22 & 147 & 107 \\
\hline Group6 & 22 & 25 & 177 & 142 \\
\hline Group7 & 25 & 28 & 207 & 158 \\
\hline Group8 & 25 & 28 & 207 & 158 \\
\hline Group9 & 28 & 31 & 267 & 181 \\
\hline Group10 & 31 & 34 & 297 & 199 \\
\hline Group11 & 34 & 37 & 327 & 211 \\
\hline Group12 & 37 & 40 & 357 & 241 \\
\hline Group13 & 40 & 43 & 387 & 262 \\
\hline Group14 & 43 & 46 & 417 & 287 \\
\hline Group15 & 46 & 49 & 447 & 310 \\
\hline
\end{tabular}

Table 2. Comparison of the Regular and Modified SSB CSLA:

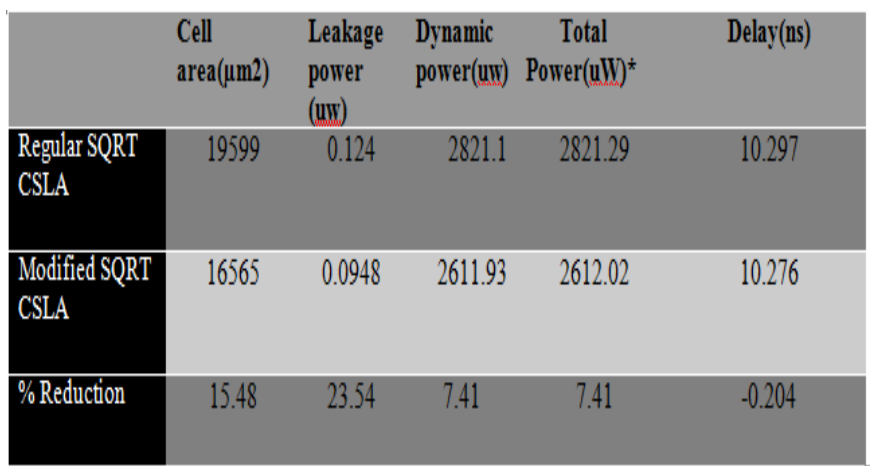

*Total power= leakage power + Internal power + Dynamic Power

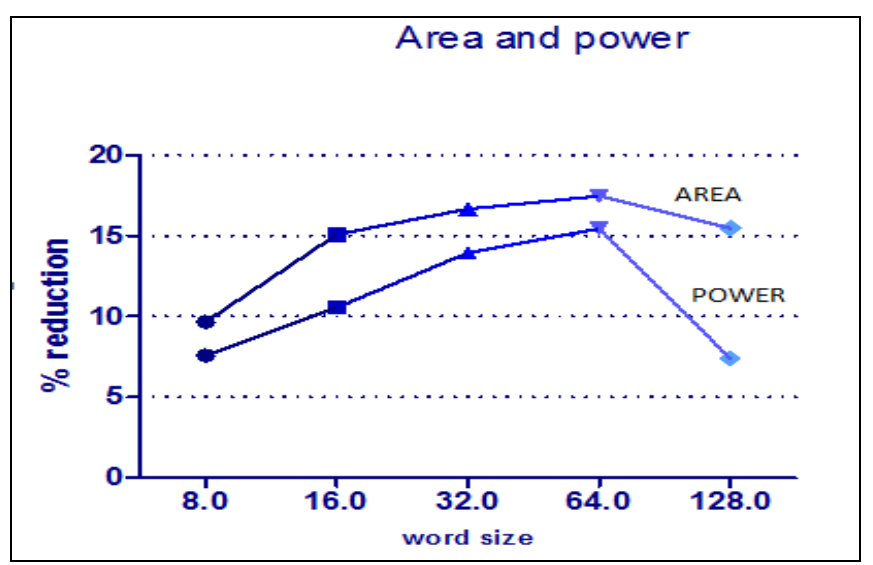

Fig.4: Percentage reduction in the cell area and total power

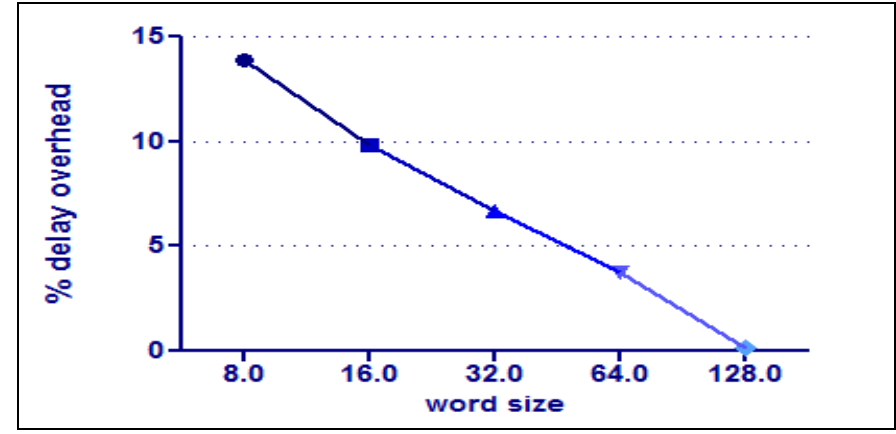

Fig.5: Percentage of delay overhead

\section{Conclusion:}

We have implemented the technique proposed in [1] for 128 bit Carry Select Adder and the reduced number of gates of this work offer great advantages in the reduction of area and also the total power. The result shows that there is a reduction of $15.48 \%$ in the cell area and the power also reduced by $7.41 \%$ with no delay ahead. The modified 128 bit CLSA is therefore low area and low power and also it is efficient for the VLSI hardware implementation. Further work can also be carried out by improve the word size to 256 word size and analyze its power, area, and delay and compare with modified version.

\section{References:}

[1] B.Ramkumar, H,Mkittur "Low -Power and Area-Efficient Carry Select Adder "I.E.E.E transaction on very large scale integration systems"

[2] B. Ramkumar, H.M. Kittur, and P. M. Kannan, "ASIC implementation of modified faster carry save adder," Eur. J Sci. Res., vol. 42, no. 1, pp.53-58, 2010

[3] Cadence, "Encounter user guide," Version 6.2.4, March 2008

[4] J. M. Rabaey, Digtal Integrated Circuits-A Design Perspective. Upper Saddle River, NJ: Prentice-Hall, 2001

[5] O. J. Bedrij, "Carry-select adder," IRE Trans. Electron. Comput., pp.340-344, 1962.

[6] T. Y. Ceiang and M. J. Hsiao, "Carry-select adder using single ripple carry adder," Electron. Lett., vol. 34, no. 22, pp. 2101-2103, Oct. 1998.

[7] Y. Kim and L.-S. Kim, "64-bit carry-select adder with reduced area,"Electron. Lett., vol. 37, no. 10, pp. 614-615, May 2001

[8] Y. He, C. H. Chang, and J. Gu, "An area efficient 64-bit square root carry-select adder for low power applications," in Proc. IEEE Int. Symp.Circuits Syst., 2005, vol. 4, pp. 4082-4085. 